\title{
Deformation behavior of extruded ZN11 magnesium plate
}

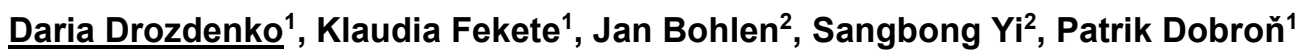

${ }^{I}$ Charles University, Faculty of Mathematics and Physics, Department of Physics of Materials, Prague, Czech Republic; ${ }^{2}$ Helmholtz-Zentrum Hereon, Institute of Material and Process Design, Department of Material Design, Geesthacht, Germany;

\section{drozdenko@karlov.mff.cuni.cz}

Deformation behavior of ZN11 magnesium alloy in a form of extruded profile has been investigated with respect to different loading directions. The samples were compressed at room temperature with a constant strain rate of $10^{-3} \mathrm{~s}^{-1}$ along extrusion (ED), transversal (TD) and normal direction (ND). X-ray diffraction technique was employed to follow the development of texture during loading. The twinning activity was studied by the subsequent analysis of microstructure using scanning electron microscopy (BSD, EBSD). The deformation behavior of the extruded profile was also investigated by the acoustic emission (AE) technique, where the AE signal analysis correlates the microstructure and the stress-time curves to the active deformation mechanisms. Compression along the ND (i.e. compression perpendicular to the basal planes) is not favorable for twinning, while during compression along the ED and TD twinning activity is observed.

Keywords: magnesium, deformation behavior, twinning, acoustic emission

The financial support by ERDF (project 'NanoCent' No. CZ.02.1.01/0.0/0.0/15 003/0000485) is gratefully acknowledged. 